\title{
Mathematic Description Method of Hybrid Electric Vehicles' Driving Cycle
}

\author{
WANG Ximing ${ }^{a}$, HE Hongwen ${ }^{b}$, LIU Xinlei ${ }^{c}$, PENG Jiankun ${ }^{d}$, TANG Henglu ${ }^{e}$ \\ National Engineering Laboratory for Electric Vehicles, School of Mechanical Engineering; Beijing \\ Institute of Technology, Beijing, China \\ E-mail: axmhn163@163.com, ${ }^{b}$ hwhebit@bit.edu.cn, ${ }^{c}$ liuxinlei1981@yahoo.cn, \\ dpeng_jiankun@163.com, ehenglutang@126.com
}

Keywords: Mathematic model; Driving cycle; Hybrid electric vehicle; Hybrid powertrain

\begin{abstract}
The performance of fuel economy and emissions reduction of hybrid electric vehicles (HEVs) strongly is affected by the target driving cycle. So, the characteristics of the driving cycle play an important role in the hybrid powertrain matching and the control strategy designing. However, the mathematic description method about the characteristics of the driving cycle has been not studied before. The mathematic model is established to describe the characteristics of the driving cycle in this paper, which provide a useful guidance to design a hybrid electric vehicle that operates efficiently under the target driving cycle.
\end{abstract}

\section{Introduction}

The problems derived from the climate change have become more and more serious, and promote the applications such technologies as saving energy and reducing emissions in the modern vehicle fast. Compared with the conventional vehicles, hybrid electric vehicles (HEVs) have been paid great attentions due to its superiority in saving energy and reducing emissions. The performance of $\mathrm{HEV}$ is largely affected by the characteristic of the target driving cycle $[1,2,3]$. So it plays an important role in the hybrid powertrain matching and the control strategy designing. However, the mathematic description method about the characteristics of the driving cycle has been not studied before. Herein, the mathematic model is established to describe the characteristics of the driving cycle.

\section{Characteristic Parameters of the Driving Cycle}

The driving cycle is the relationship between the desired velocity and the time which reflects the demand power, the demand torque and the demand speed. The basic characteristics of the driving cycle include the maximum velocity, the average velocity, the average propelling velocity, the idle ratio, the constant-velocity ratio, the acceleration ratio and the deceleration ratio. While its power characteristics are embodied by the average propelling demand power, the maximum propelling demand power, the maximum speed, the average braking demand power, the maximum braking demand power, the maximum propelling demand torque and the maximum braking demand torque, which represent the individual demands to hybrid powertrain.

\section{Driving Cycle's Mathematic Model}

Mathematic Description of Basic Characteristics

The maximum velocity of the driving cycle is described in Eq.1.

$$
u_{a_{-} c y c_{-} \max }=\max \left(u_{a_{-} c y c_{-} t}\right)
$$

Where $u_{a_{-} c y c_{-} \max }$ is the maximum velocity; $u_{a_{-} c y c_{-} t}$ is the instantaneous velocity at the $t$ moment in the driving cycle.

The average velocity of the driving cycle is described in Eq.2. 


$$
\bar{u}_{a_{-} c y c}=\frac{S_{c y c}}{T_{c y c}}
$$

Where $\bar{u}_{a_{-} c y c}$ is the average velocity; $S_{c y c}$ is the total mileage of the driving cycle, which is given by Eq.3; $T_{c y c}$ is the total duration time of the driving cycle.

$$
S_{c y c}=\sum_{j=1}^{l} u_{a_{-} c y c_{-} j} \cdot d t
$$

Where $l$ is sampling numbers in the driving status of the target driving cycle, which is given by Eq.4; $j$ is the sampling point; $u_{a_{-} c y c_{-} j}$ is the velocity at the sampling point of $j ; d t$ is the sampling period.

$$
l=\frac{T_{c y c_{-} d}}{d t}
$$

Where $T_{c y c \_d}$ is the total propelling period of the driving cycle.

The average velocity in the propelling status of the driving cycle is described in Eq.5.

$$
\bar{u}_{a_{-} c y c_{-} d}=\frac{S_{c y c}}{T_{c y c_{-} d}} \times 100 \%
$$

Where $\bar{u}_{a_{-} c y c_{-} d}$ is the average propelling velocity; $T_{c y c_{-} d}$ is propelling period of the driving cycle.

The idle ratio of the driving cycle is described in Eq.6.

$$
K_{c y c_{-} i d l e}=\frac{T_{c y c_{-} i d l e}}{T_{c y c}} \times 100 \%
$$

Where $K_{\text {cyc_idle }}$ is the idle ratio; $T_{\text {cyc_idle }}$ is idle period of the driving cycle.

The constant-velocity ratio of the driving cycle is described in Eq.7.

$$
K_{c y c_{-} \text {con }}=\frac{T_{c y c_{-} c o n}}{T_{c y c}} \times 100 \%
$$

Where $K_{c y c \_c o n}$ is the constant-velocity ratio; $T_{c y c_{-} c o n}$ is constant-velocity period of the driving cycle.

The acceleration ratio of the driving cycle is described in Eq.8.

$$
K_{c y c_{\_} a c c}=\frac{T_{c y c_{-} a c c}}{T_{c y c}} \times 100 \%
$$

Where $K_{\text {cyc_acc }}$ is the acceleration ratio; $T_{\text {cyc_acc }}$ is acceleration period of the driving cycle.

The deceleration ratio of the driving cycle is described in Eq.9.

$$
K_{c y c \_d e c}=\frac{T_{c y c \_d e c}}{T_{c y c}} \times 100 \%
$$

Where $K_{\text {cyc_dec }}$ is the deceleration ratio; $T_{\text {cyc_dec }}$ is deceleration period of the driving cycle.

\section{Mathematic Description of Power Characteristics}

The maximum propelling demand power, the maximum propelling demand torque and the maximum demand speed represent the maximum demands of the driving cycle to the propelling ability of the hybrid powertrain, which are respectively described by Eq.10, Eq.13 and Eq.16. The maximum braking demand power and the maximum braking demand torque represent the demands of the driving cycle to the regenerating ability of the hybrid powertrain, which are respectively described by Eq.18 and Eq.20.

$$
P_{c y c_{-} d_{-} \max }=\max \left(P_{c y c_{-} d_{-} t}\right)
$$

Where $P_{c y c_{-} d_{-} \max }$ is the maximum propelling power; $P_{c y c_{-} d_{-} t}$ is the instantaneous propelling 
power, which is given by Eq.11.

$$
P_{c y c_{-} d_{-} t}=\left\{\begin{array}{cl}
P_{c y c_{-} t} & , P_{c y c_{\_} t} \geq 0 \\
0 & , P_{c y c_{-} t}<0
\end{array}\right.
$$

Where $P_{\text {cyc_t }}$ is the instantaneous demand power, which is given by Eq.12.

$$
P_{c y c_{-} t}=\frac{1}{3600}\left(m g f+\frac{C_{D} \cdot A \cdot u_{a_{-} c y c_{-} t}{ }^{2}}{21.15}+m \delta \cdot \frac{u_{a_{-} c y c_{-} t}-u_{a_{-} c y c_{-} t-1}}{3.6 \cdot d t}\right) \cdot u_{a_{-} c y c_{-} t}
$$

Where $m$ is the vehicle gross mass; $g$ is the gravitational acceleration; $f$ is the rolling resistance coefficient; $C_{D}$ is the aerodynamic drag coefficient; $A$ is the vehicle frontal area; $u_{a_{-} c y c_{-} t-1}$ is the instantaneous velocity at the $t-1$ moment in the driving cycle. $\delta$ is the vehicle rotational inertial factor, which is the constant to be 1.1 here.

$$
T_{c y c_{-} d_{-} \max }=\max \left(T_{c y c_{-} d_{-} t}\right)
$$

Where $T_{c y c_{-} d_{-} \max }$ is the maximum propelling torque; $T_{c y c_{-} d_{-} t}$ is the instantaneous propelling torque, which is given by Eq.14.

$$
T_{c y c_{-} d_{-} t}=\left\{\begin{array}{cc}
T_{c y c_{-} t} & , T_{c y c_{-} t} \geq 0 \\
0 & , T_{c y c_{-} t}<0
\end{array}\right.
$$

Where $T_{\text {cyc } t}$ is the instantaneous demand torque, which is given by Eq.15.

$$
T_{c y c_{-} t}=r_{d} \cdot\left(m g f+\frac{C_{D} \cdot A \cdot u_{a_{-} c y c_{-} t}^{2}}{21.15}+m \delta \cdot \frac{u_{a_{-} c y c_{-} t}-u_{a_{-} c y c_{-} t-1}}{3.6 \cdot d t}\right)
$$

Where $r_{d}$ is the dynamic radius of the wheel.

$$
n_{c y c_{-} \max }=\max \left(n_{\text {cyc_t }}\right)
$$

Where $n_{c y c_{-} \max }$ is the maximum speed; $n_{c y c_{-} t}$ is the instantaneous speed, which is given by Eq.17 [4].

$$
\begin{gathered}
n_{c y c_{-} t}=\frac{1000 \cdot u_{a_{-} c y c_{-} t}}{2 \pi r_{d} \cdot 60} \\
P_{c y c_{-} b_{-} \max }=\left|\min \left(P_{c y c_{-} b_{-} t}\right)\right|
\end{gathered}
$$

Where $P_{c y c_{-} b_{-} \max }$ is the maximum braking power; $P_{c y c_{-} b_{-} t}$ is the instantaneous braking power, which is given by Eq.19.

$$
\begin{gathered}
P_{c y c_{-} b_{-} t}=\left\{\begin{array}{cc}
0 & , P_{c y c_{-} t} \geq 0 \\
P_{c y c_{-} t} & , P_{c y c_{-} t}<0
\end{array}\right. \\
T_{c y c_{-} b_{-} \max }=\left|\min \left(T_{c y c_{-} b_{-} t}\right)\right|
\end{gathered}
$$

Where $T_{c y c_{-} b_{-} \max }$ is the maximum braking torque; $T_{c y c_{-} b_{-} t}$ is the instantaneous braking torque, which is given by Eq.21.

$$
T_{c y c_{-} b_{-} t}=\left\{\begin{array}{cc}
0 & , T_{c y c_{-} t} \geq 0 \\
T_{c y c_{-} t} & , T_{c y c_{-} t}<0
\end{array}\right.
$$

Moreover, the average demand power in the propelling status of the driving cycle represents the average demand power of the driving cycle to the propelling ability of the hybrid powertrain, which is described in Eq.22, and the average braking demand power represents the average demand power to the regenerating ability, which is described in Eq.23.

$$
\bar{P}_{c y c_{-} d}=\frac{\sum_{j=1}^{l} P_{c y c_{-} d_{-} j} \cdot d t}{T_{c y c_{-} d}}
$$


Where $\bar{P}_{c y c \_d}$ is the average demand power in the driving status of the target driving cycle; $P_{c y c_{-} d_{-} j}$ is the propelling demand power at the sampling point of $j$.

$$
\bar{P}_{c y c_{-} \mathrm{b}}=\frac{\sum_{j=1}^{l} P_{c y c_{-} b_{-} j} \cdot d t}{T_{c y c_{-} b}}
$$

Where $\bar{P}_{c y c \_ \text {b }}$ is the average demand power in the braking status of the target driving cycle; $P_{c y c_{-} b_{-} j}$ is the braking demand power at the sampling point of $j ; T_{c y c_{-} d}$ is the total braking period of the driving cycle.

\section{Analysis Example}

A hybrid electric bus and the standard urban driving cycle in China [5] are selected to illustrate. The vehicle parameters of the selected bus are listed in Table 1, and the profiles of the selected driving cycle is shown in Fig.1.

Table 1 Vehicle parameters of the hybrid electric bus

\begin{tabular}{cccc}
\hline Parameters & Values & Parameters & Values \\
\hline Curb mass $[\mathrm{kg}]$ & 11000 & Air drag coefficient & 0.55 \\
Gross mass $[\mathrm{kg}]$ & 17800 & Rolling resistance coefficient & 0.0057 \\
Vehicle frontal area $\left[\mathrm{m}^{2}\right]$ & 6.6 & Dynamic radius of the wheel $[\mathrm{mm}]$ & 473 \\
\hline
\end{tabular}

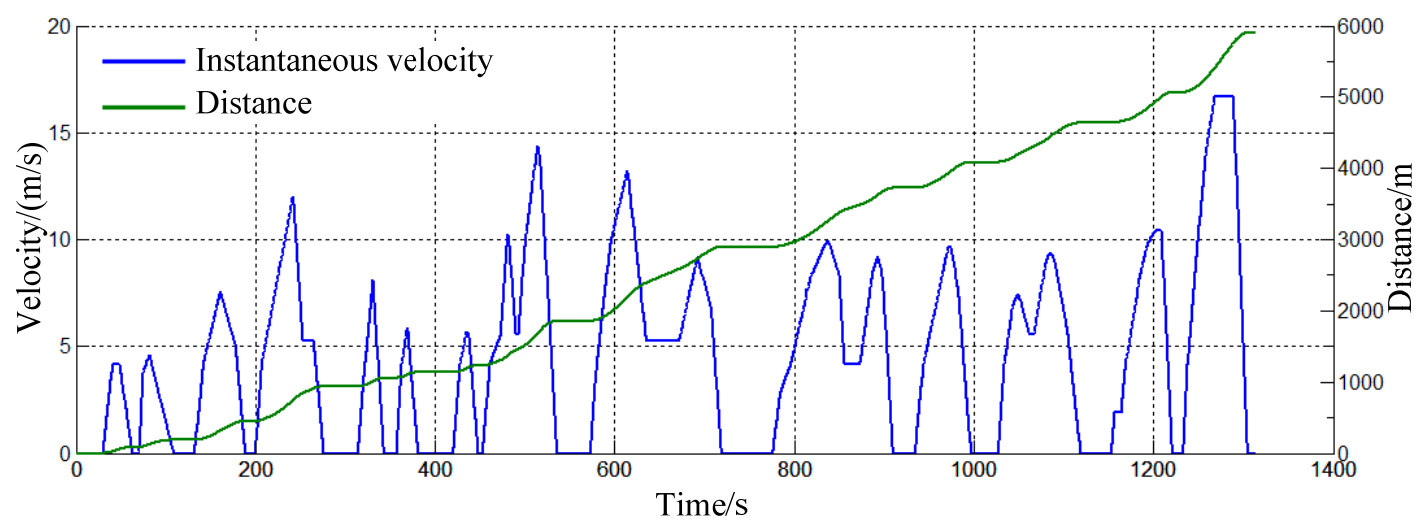

Fig.2 Profiles of the velocity and the travel distance of the standard urban driving cycle in China

According to the mathematic description method presented before, the basic characteristics and the power characteristics of the selected driving cycle are listed in Table 2.

Table 2 Characteristic parameters of the standard urban driving cycle in China

\begin{tabular}{cccc}
\hline Parameters & Values & Parameters & Values \\
\hline Average velocity [km/h] & 15.9 & Deceleration ratio [\%] & 25.46 \\
Maximum velocity [km/h] & 60.0 & Maximum propelling demand power [kW] & 143.21 \\
Average propelling velocity & 32.08 & Maximum braking demand power [kW] & 303.88 \\
{$[\mathrm{~km} / \mathrm{h}]$} & 5.8 & Maximum propelling demand torque [Nm] & 8938.00 \\
Total mileage[km] & 1314 & Maximum braking demand torque [Nm] & 9177.00 \\
Total duration time [s] & 28.58 & Maximum demand speed [r/min] & 336.50 \\
Idle ratio[\%] & 8.75 & Average propelling demand power [kW] & 34.32 \\
Constant-velocity ratio[\%] & Average braking demand power [kW] & 41.23 \\
Acceleration ratio [\%] & 37.25 & &
\end{tabular}


The instantaneous characteristics of the power demands are shown in Fig.3, Fig.4 and Fig.5.

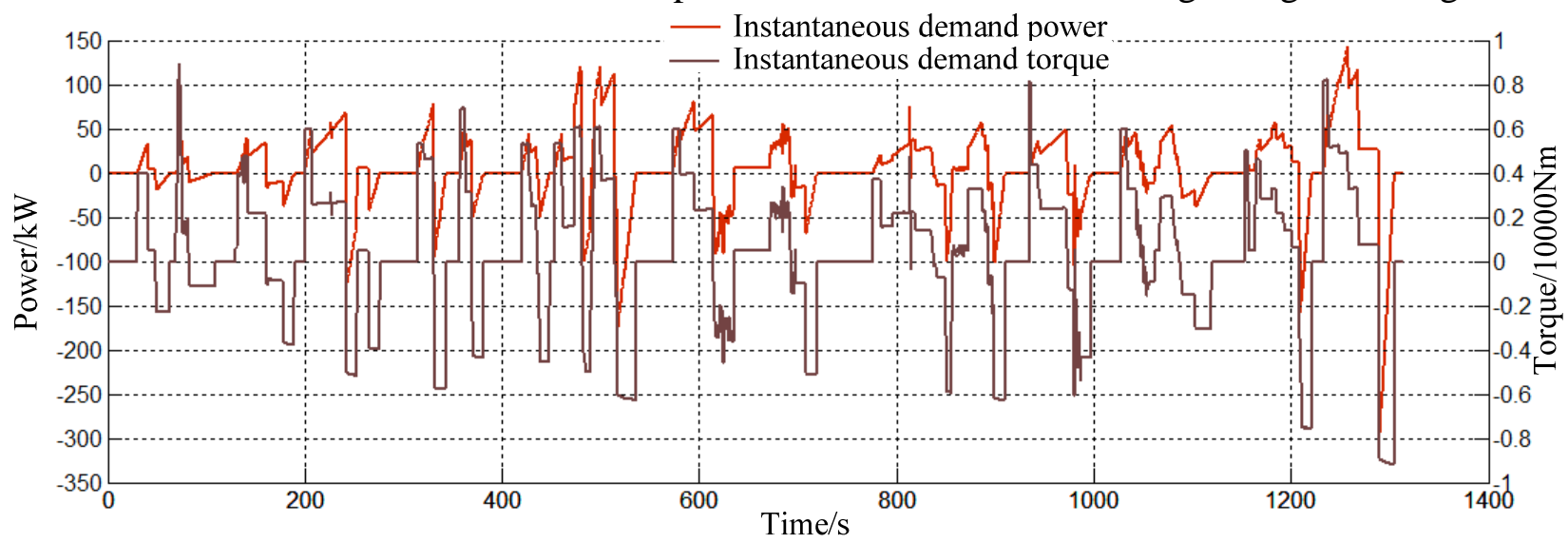

Fig.2 Instantaneous characteristics of the demand power and the demand torque of the standard urban driving cycle in China

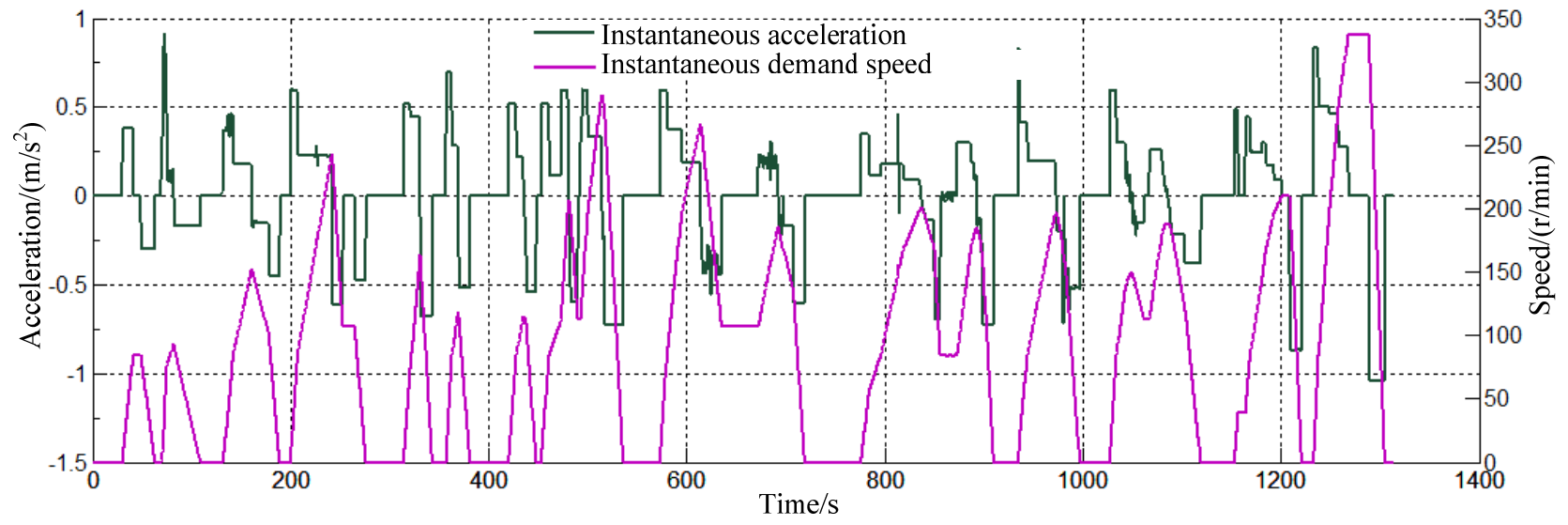

Fig.3 Instantaneous characteristics of the acceleration and the demand speed of the standard urban driving cycle in China

\section{Summary}

In this paper, the mathematic model is established to describe the characteristics of the driving cycle which include the basic characteristics and the power characteristics. It could provide a useful guidance in matching hybrid powertrain and designing control strategy, so that it is possible to design a hybrid electric vehicle that operates more efficiently under the target driving cycle.

\section{Reference}

[1] Luo Yu-tao, Hu Hong-fei, Shen Ji-jun. Analysis and Recognization of Running Cycles of Hybrid Electric Vehicle [J]. Journal of South China University of Technology (Natural Science Edition), 2007,35(6):8-13.

[2] Wang Wei, Wang Qing-nian, Wang Peng-yu, et al. Rated power and efficiency matching of induction motor for parallel hybrid electric vehicle based on vehicle drive cycle [J]. Journal of Jilin University (Engineering and Technology Edition), 2008,38(supplement):12-17.

[3] Wang Baohua, Luo Yongge. Simulation on Performance of City Bus Based on Typical Urban Driving Cycle [J]. Journal of Hubei Automotive Industries Institute, 2007, 21(1): 6-9,13.

[4] Yu Zhisheng. Automotive theory [M]. Beijing: Mechanical Engineering Press.

[5] GB/T19754-2005, Measurement Method of the Energy Consumption on the Heavy Duty Hybrid Electric Vehicle [S]. Beijing: China Standards Press, 2005. 